\title{
Compact Microwave Cavity with Increased Magnetic Field Homogeneity
}

\author{
Anton E. Ivanov ${ }^{1}$, Anja K. Skrivervik ${ }^{1}$, Christoph Affolderbach ${ }^{2}$, Gaetano Mileti ${ }^{2}$ \\ ${ }^{1}$ Laboratoire d'Électromagnétisme et d'Acoustique (LEMA), École Polytechnique Fédérale de Lausanne (EPFL), \\ Lausanne, Switzerland, E-mail: antonevgeniev.ivanov@epfl.ch \\ ${ }^{2}$ Laboratoire Temps-Fréquence, University of Neuchâtel, Neuchâtel, Switzerland
}

\begin{abstract}
In this study we aim at improving the homogeneity of the magnetic field inside a microwave resonator cavity designed in line with the development of a novel, highperformance frequency standard. We study the effect of modified boundary conditions applied to a cavity based on a loop-gap geometry, and demonstrate the high potential of this cavity to improve the frequency stability. Our concept is suitable for the future generation of compact, high-precision frequency standards based on vapor cells and pulsed optical pumping regime (POP atomic clocks). These novel clocks are excellent candidate for future applications in space or mobile applications where highly compact instruments are required.
\end{abstract}

Index Terms-microwave cavities, magnetic field homogeneity, frequency standards, atomic clocks.

\section{INTRODUCTION}

In general, frequency standards based on vapor cells and a double resonance scheme [1] are good solutions for applications that require excellent frequency stability. In terms of performance they are situated between the largescale laboratory devices [2] or commercial Cs clocks [3] and the quartz oscillators widely employed in end user products [4]. Lately a new generation of vapor-cell clocks based on pulsed optical pumping (POP) proved to have promising performance with state-of-the-art long-term stability [5], currently from a still rather big laboratory demonstrator. However, for a pulsed clock, the magnetic field homogeneity has a central role limiting the clock performance [6], which makes the design of the cavity one of the crucial components of the clock. In this study we investigate the electromagnetic problem and propose a solution based on a loop-gap cavity and artificial boundary conditions.

\section{VAPOR CELL FREQUENCY STANDARDS}

A highly stable frequency signal is possible if the oscillator is based on a physical process that is ideally independent on perturbations from the external world. For example a commercial, temperature-stabilized, quartz oscillator is able to provide frequency stability, measured in terms of Allan deviation, of about $1 \times 10^{-12} \tau^{-1 / 2}$ for integration times of 1-100 s (short-term stability). Since these oscillators are highly susceptible to changes in temperate and mechanical vibrations, their performance does not allow for highly demanding applications (ex. the synchronization of GPS satellites requires stable on-board frequency with the stability being a limitation for the resolution of the GPS positioning). The solution is to lock the quartz oscillator to another, more stable process based on atomic resonance. Among the large family of such high-performance frequency standards the vapor cell atomic clock based on double resonance (DR) and rubidium atoms is a very compact and relatively low-cost device suitable for various applications in communication, future smart grids and satellite navigation [7].

\section{A. Pulsed Clock Operation}

Such a clock consists of a collection of atoms that can be considered as very stable oscillators, coherently driven by an external field. Each atom can be seen as a simplified threelevel system (figure 1).

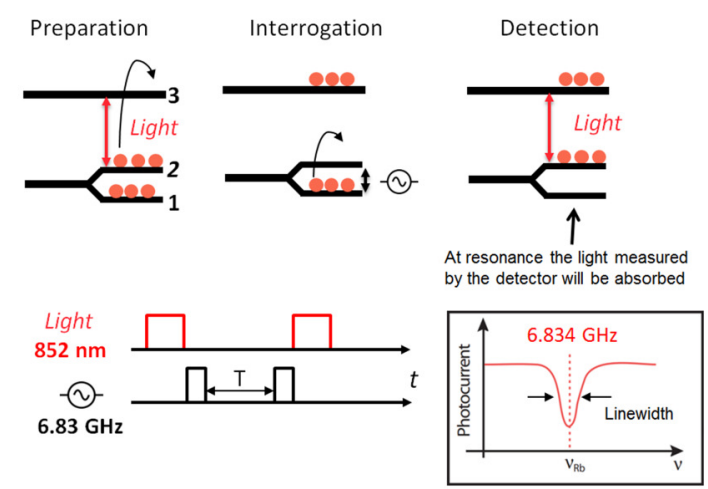

Fig. 1. Atomic resonance in the ${ }^{87} \mathrm{Rb}$ atom applied in the pulsed regime. The atoms are represented by a three-level system. The oscillator is created by cycles of absorption and emission between levels 1-2 and is driven by magnetic field tuned to the frequency of the transition $-6.83 \mathrm{GHz}$. The oscillation signal can be distinguished only when there is a population imbalance (population inversion) between the levels 1-2. Laser light tuned to the transition 2-3 is both used to create the required population inversion and to detect the resonance.

The operation of the pulsed clock is divided into three different stages separated in time. Initially, without any external field applied, the rubidium atoms will have electrons populating levels 1 and 2 . In the preparation stage, a pulse of 
laser light tuned to the energy difference between levels 2 and 3 is applied. After all the atoms are excited and level 2 is "empty", the laser is switched off and a microwave magnetic field with frequency $6.834 \mathrm{GHz}$ is applied to the atoms (figure 1 - interrogation). In the presence of this external field the atoms can be classically seen as magnetic dipoles oriented in opposite directions according to the populated level. Only when the microwave frequency is exactly tuned to the difference between levels 1 and 2 the electrons will populate the empty level 2 ( $180^{\circ}$ flip of the magnetic dipoles). Therefore, if a laser pulse of light is applied again, large amount of light photons will be absorbed by the electrons that now populate level 2, (figure 1 - detection). Therefore, by cycles of sweeping the microwave magnetic field and measuring the amount of absorbed light we can constantly lock the frequency of the input signal to the magnetic resonance line, for which we observe the absorption of the laser light. Because the energy levels are in reality degenerate, the exploited magnetic resonance transition will be welldefined only when an additional static magnetic field (C-field) is applied to the atoms - Zeeman splitting. The aboveexplained interaction of the atoms with the external fields is physically realized in the physics package of the clock (figure 2).

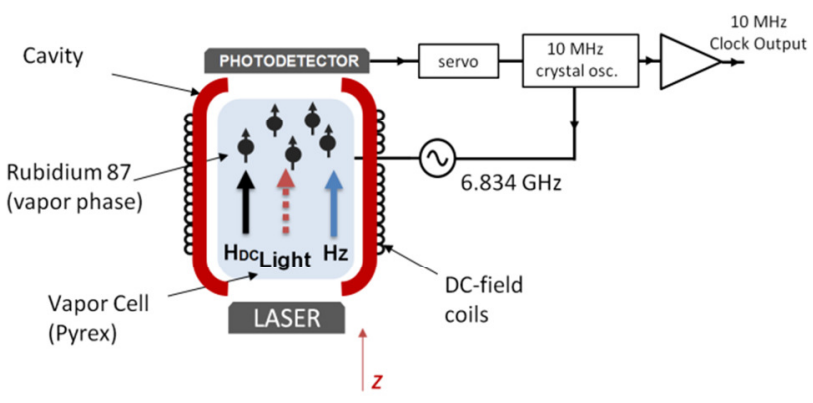

Fig. 2. The rubidium atoms in vapor phase are enclosed in a dielectric cell (Pyrex) situated inside the microwave cavity. Two large openings allow the laser light to penetrate, interact with the atoms and get detected by the photodetector. The $\mathrm{C}$-field required for the Zeeman splitting is created by the coils surrounding the cavity and is oriented in the z-direction shown.

We note that in a traditional vapor cell clock the operation is implemented in a continuous mode - the laser light and the microwave fields are not applied separately. Because the light perturbs the magnetic resonance condition, instability of the laser will be translated to the magnetic resonance condition and will thus degrade the stability [7]. In POP clocks however, this is eliminated by the temporal separation between the two fields, allowing for improved frequency stability.

\section{B. Cavity Design Requirements}

Traditionally circular cavities are preferred because they can be easily optimized to enclose the vapor cells and to hold the coils required for the $\mathrm{C}$-field. The most important requirement that applies to the cavity is to provide all atoms with spatially well-defined microwave fields - only the magnetic field component aligned to the direction of the $\mathrm{C}$-field is resonant to the selected transition (at $6.834 \mathrm{GHz}$ ) and will contribute to the beneficial signal. Therefore a TE mode is the proper choice, resulting in a large amount of $\mathrm{H}_{\mathrm{z}}$ magnetic field aligned to both the C-field and the laser light (figure 2). We note that the transverse magnetic field components of the cavity are highly undesirable since they will drive resonances that are unwanted, leading to a reduction of the clock's frequency stability. The ability of the cavity to provide the beneficial field can be described by a figure of merit, Field Orientation Factor - FOF defined as [8]:

$$
\xi=\frac{\int_{\text {Vcell }} H_{z}{ }^{2} d V}{\int_{\text {Vcell }}|\mathbf{H}|^{2} d V},
$$

where $\mathrm{H}_{\mathrm{z}}$ is the beneficial magnetic field component aligned to the direction of the $\mathbf{C}$-field, and $\mathbf{H}$ is the total magnetic field. Fundamentally, the frequency stability of such an oscillator can be related to the Q-factor and the signal-to-noise ratio by [9]:

$$
\sigma=\frac{0.2}{Q .(S / N)} \tau^{-1 / 2}
$$

Where $\sigma$ is the standard Allan deviation defined for integration time $\tau$. Thus, via the design of the cavity we can improve the frequency stability by two major ways:

- If the cavity is able to accommodate enlarged vapor cells, higher amount of $\mathrm{Rb}$ atoms will absorb more light photons thus improve the signal-to-noise ratio in the detected signal. Larger openings for the laser light are favorable for the same reason.

- Inside the cavity, the spatial distribution is such that not all atoms will interact with the same field amplitude of $\mathrm{H}_{\mathrm{z}}$. Seen as a collection of magnetic dipoles, depending on the amplitude of $\mathrm{H}_{\mathrm{z}}$, their magnetic moments will "flip" more or less than the required for resonance $180^{\circ}$. Macroscopically this effect will be seen as damping of the atomic oscillator, and it will broaden the resonance line observed at the photodetector (figure 1). Therefore, increasing the Q-factor of the atomic resonance (different from the Q-factor of the cavity) is possible if inside the active zone of the cavity the spatial variation is reduced for $\mathrm{H}_{\mathrm{z}}$.

\section{MAGNETRON-TYPE CAVITY}

The circular cavity (cylinder with circular cross section) is a practical and common choice for vapor cell clocks [10]. If the cell is situated at its center, the TE011 mode provides a microwave magnetic field that can interact with enough number of atoms. When compared, the volume of the cavity is a few times larger than the volume of the vapor cell [11]. This inherent limitation of the circular geometry is avoided for a 
cavity based on a magnetron-type structure (figure 3). In the literature the latter is also known as split-ring and loop-gap resonator [12]. It consists of a cylindrical volume filled with metal electrodes which form a combination of capacitive and inductive behavior. We have previously designed such a magnetron-type cavity able to hold an enlarged cell of $25 \mathrm{~mm}$ in diameter being still more compact than other geometries. This is due to the high field orientation factor [8] - more than $85 \%$ of the total magnetic field inside the magnetron cavity is oriented along the required direction of the $\mathrm{C}$-field. The shortterm stability of a clock based on this cavity was reported to be $1.4 \times 10^{-13} \tau^{-1 / 2}$ in continuous interrogation mode [13].

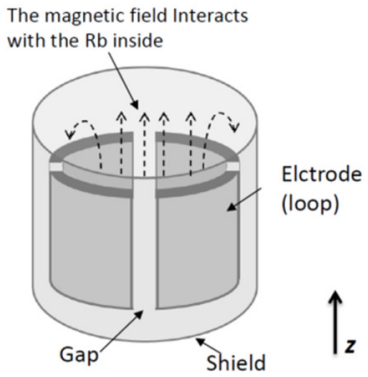

Fig. 3. Simplified scheme of cavity based on magnetron (loop-gap) geometry.

\section{MAGNETIC FIELD DistribUtion}

The spatial variation of the field inside the cavity is the cumulative effect of the standing wave distribution in the transverse and longitudinal direction (along $\mathrm{z}$ ). The former depends on the shape of the chosen cross section while the latter is described by a sinusoidal variation. Therefore, in order to increase the field homogeneity inside the resonator we would like to keep the modal indexes $\left(\mathrm{TE}_{\mathrm{mnl}}\right)$ as close to zero as possible, while still be able to maintain the required $\mathrm{H}_{\mathrm{z}}$ field at the frequency of interest. In the current study we aim at minimizing the sinusoidal variation of the field in the $\mathrm{z}$ direction and thus significantly improve the total field homogeneity inside the cavity. It is easy to see that for the mode of interest such requirements cannot be fulfilled by applying PEC boundary conditions since the $\mathrm{H}_{\mathrm{z}}$ component is always normal to the boundary and therefore zero at each of the two end planes. It is however possible to exploit the complementary situation of PEC - i.e. the perfect magnetic conductor (PMC) boundary condition. In the circular cavity, one possible way to create boundary conditions that mimic PMC was extensively studied in [14] and is shown in Figure 4. Physically, at the cutoff limit the guided wave is characterized by an infinite wavelength, therefore a zero variation of the field along the propagation axis and longitudinal wavenumber zero. Therefore when the cavity is designed with symmetrical dielectric segments both having length defined as:

$$
a=\frac{\sqrt{\mu_{0} \varepsilon_{0}}}{4 f_{\text {cutoff }}} \frac{1}{\sqrt{\left(\varepsilon_{r}-1\right)}},
$$

which corresponds to $1 / 4$ of the wavelength inside the waveguide segment filled with dielectric with relative permittivity $\varepsilon_{r}$. At the cutoff frequency they can be seen as PMC boundary conditions allowing nearly constant field distribution inside the central part.
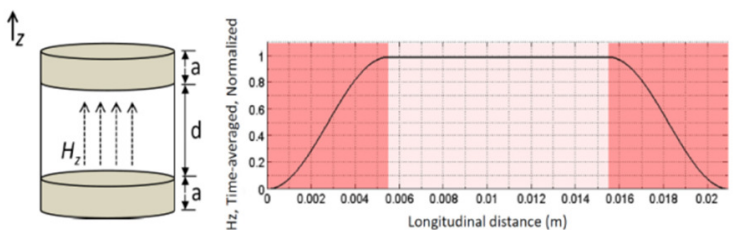

Fig. 4. (Left) - Fully enclosed circular cavity with artificial boundary conditions. The two top and bottom segments (length a) are homogeneously filled with dielectric. (Right) $-\mathrm{H}_{\mathrm{z}}$ component along the $\mathrm{z}$ axis of the cavity. In the middle - constant field region, the two dielectric segments are shown in red. The cutoff frequency $f_{\text {cutoff }}=6.835 \mathrm{GHz}$ corresponds to the proper clock transition. The cavity is $0.1 \%$ above cutoff. The length of the two dielectric layers is $\mathrm{a}=5.5 \mathrm{~mm}$ and it was calculated for Pyrex.

We propose a simple figure of merit that enables the evaluation of the total field homogeneity inside a cavity field homogeneity histogram. In figure 5 we show the amount of field homogeneity in a cavity with the modified boundary conditions compared to the empty one.

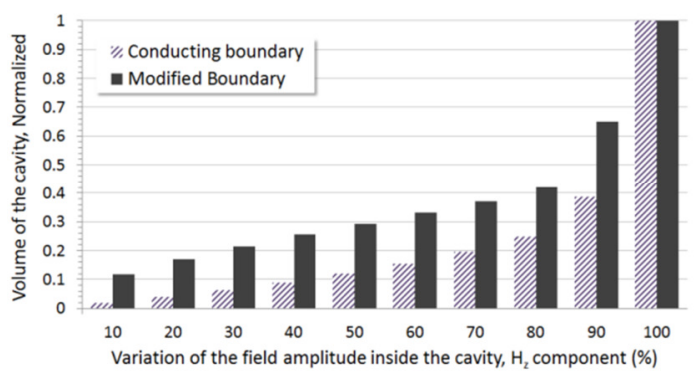

Fig. 5. Field homogeneity histogram. The magnetic field was calculated for a large set of equally distributed points inside the volume of each cavity. The obtained distribution of field amplitudes is then normalized with respect to the maximum. We define discrete intervals for the variation of the field amplitude in the range from $\quad 0-100 \%$. By comparing the number of points that have amplitude variation in a given interval to the total number of points in the set we can calculate what relative part of the volume inside the cavity "sees" field in a given variation range.

It can be concluded that by modifying the boundary conditions in the above described way, significant improvement of the field homogeneity is possible. In the following chapter we relate this approach to our loop-gap geometry. 


\section{LOOP - GAP GEOMETRY WITH MODIFIED BOUNDARY CONDITIONS}

Our magnetron cavity is shown in figure 6. The loop-gap structure consists of six metallic electrodes separated symmetrically and enclosed in a cylindrical shell. The cell containing the rubidium vapor is situated in the center, fixed to the upper cap. The latter has also a function as a tuning mechanism.

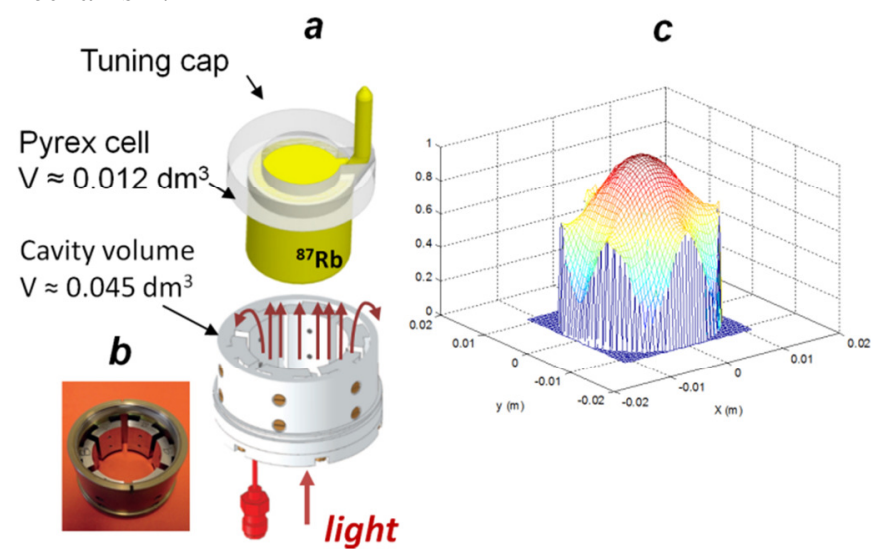

Fig. 6. Scheme of the magnetron cavity based on a loop-gap structure (a). Prototype realization (b). Amplitude profile of the $\mathrm{H}_{\mathrm{z}}$ field integrated along the height of the cavity (c).

By changing the gap size of the electrodes, the balance between the electric and magnetic energy is influenced. Therefore we can obtain the required resonance for a reduced size of the diameter as compared to the empty circular cavity. The transverse variation of $\mathrm{H}_{\mathrm{z}}$ is similar to the Bessel profile, but is peaked in the vicinity of the electrodes (figure 6-c) resulting in a good transverse homogeneity. The latter was also confirmed in a field imaging experiment [15]. In order to model the modified boundary conditions in the case of the relatively complex loop-gap geometry, we have considered a simplified transmission-line (TL) model (figure 7).

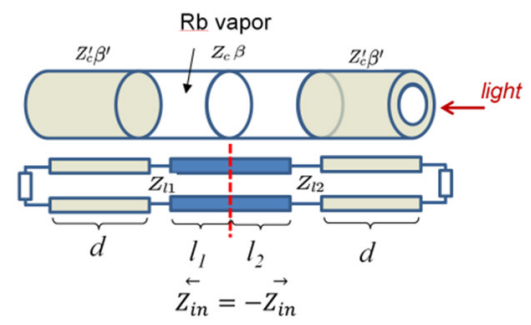

Fig. 7. Cavity with modified boundary conditions represented by a transmission line model. The resonance condition can be expressed via the impedance equality defined along the line. In the central region the $\mathrm{H}_{\mathrm{z}}$ field is constant at the cutoff frequency, thus $\beta \rightarrow 0$. This condition is fulfilled for a specific distance $d$ of the dielectric segments that can be numerically obtained from the impedance relation.

A full-wave numerical simulation performed in ANSYS high frequency simulation software (HFSS) was used to model the cavity and to obtain the parameters required for the TL model. Both the simulation and the TL model were used iteratively in order to accelerate the design procedure and gain physical understanding. In this way we were able to implement the PMC boundary conditions in two ways: via dielectric caps and alternatively by using perturbation rings figure 8 .

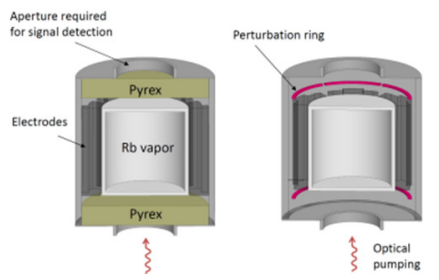

Fig. 8. Scheme of the magnetron cavity based on a loop-gap structure and two implementations of PMC boundary conditions: using transverse Pyrex caps (left) and perturbation rings marked with red (right).

The implementation based on dielectric caps can be applied if the dielectric is chosen to be transparent for the laser light (ex. Pyrex). We note that in the limit case the structure will have a high geometrical sensitivity. Furthermore a mechanism for tuning is difficult to realize since, in principal, the mode is invariant in the longitudinal direction. Therefore, although simple, the first solution is not flexible enough. In order to overcome these limitations, we have considered an alternative way to implement PMC boundary conditions similar to [16]. Because the PMC condition can be seen as a parallel LC circuit at resonance, it can be realized by perturbing the fringing fields at the end of the loop-gap region until a balance for the electric/magnetic energy is obtained. Given the mode, the fringing field in the vicinity of the openings is mostly magnetic and therefore need to be compensated with a perturbation structure that is able to provide enough electric energy. Our idea is to use movable thin metal rings on both sides of the loop-gap region (figure 9). They are very thin and light, can be implemented using planar technology and have minimal losses. The initial studies show that by changing the position of the ring with a realistic precision, about $5 \%$ relative difference between the electric and magnetic energy can be obtained corresponding to resonance frequency of $\sim 3 \%$ above the cutoff frequency.

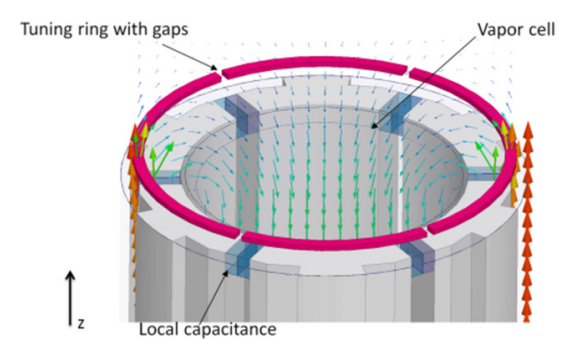

Fig. 9. Tuning ring used to implement the PMC boundary condition. The lines of the magnetic field are shown on the scheme. In order to increase the electric energy, gaps are considered for the tuning ring and dielectric material is locally introduced between the electrodes of the cavity. 
Finally we compare the obtained longitudinal field variation (along z) that will be "seen" by the atoms in the vapor cellfigure 10 .

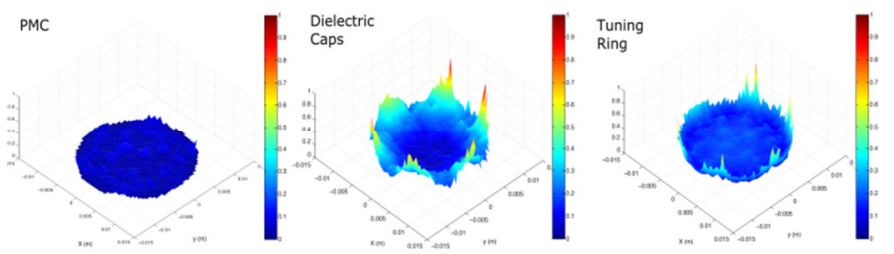

Fig. 10. Variation maps obtained for the longitudinal distribution of the $\mathrm{H}_{z}$ field in a cavity modeled with ideal PMC compared to the two implementations of PMC. Zero variation corresponds to constant field along $\mathrm{z}$.

As expected, the magnetic field variation along the $\mathrm{z}$ axis is close to zero everywhere in the cavity for which the boundary conditions are modelled as ideal PMC. The homogeneity for the cavity with the dielectric caps is very good in the center (for radius of $5 \mathrm{~mm}$ less than $10 \%$ variation), but degrades in the vicinity of the electrodes. In the case of PMC realized via the tuning ring, the variation is in the $20 \%$ range but is almost constant across the whole cross section of the vapor cell.

\section{CONCLUSION}

In this study we have identified the general EM problem and the design requirements of a of state-of-the-art vapor cell clock. We show that a solution combining a loop-gap geometry and implementation of the PMC boundary conditions can significantly increase the field homogeneity. The main design challenges were discussed and realization that allows for tenability was proposed. Such a compact cavity meets the strict design requirements and allows for a reduction of the clock instrument size while maintaining the excellent clock stability.

\section{REFERENCES}

[1] J. Vanier and C. Mandache, "The passive optically pumped $\mathrm{Rb}$ frequency standard: the laser approach", Appl. Phys. B 87, pp. 565-593, 2007.

[2] R. Wynands and S. Weyers, "Atomic fountain clocks", Metrologia 42 pp S64-S79, 2005.

[3] Le. S. Cutler, "Fifty years of commercial caesium clocks", Metrologia 42, pp S90-S99, 2005.

[4] J. R. Vig, "Quartz crystal resonators and oscilators for frequency control and timing applications - a tutorial", Rev. 8.5.1.2, 2001.

[5] S. Micalizio, C. E. Calosso, A. Godone and F. Levi, "Metrological characterization of the pulsed Rb clock with optical detection", Metrologia 49, pp. 425-436, 2012.

[6] S. Micalizio, A. Godone, F. Levi and C. Calosso, "Pulsed optically pumped Rb87 vapor cell frequency standard: A multilevel approach," Phys. Rev. A 79, 013403, 2009.

[7] J. Camparo, Phys. Today 60, 33, 2007.

[8] C. Stefanucci, T. Bandi, F. Merli, M. Pellaton, C. Affolderbach, G. Mileti, and A. K. Skrivervik, "Compact microwave cavity for high performance rubidium frequency standards". Rev. Sci. Instrum., vol. 83, 104706, Oct. 2012.
[9] J. Vanier, L. Bernier, IEEE Trans. on Instr. and Meas., Vol. IM - 30, No 4, 1981.

[10] J. Vanier, C. Audoin, "The quantum physics of atomic frequency standards", (Adam Hilger, 1989)

[11] A. Godone, S. Micalizio, F. Levi, and C. Calosso, "Microwave cavities for vapor cell frequency standards", Rev. Sci. Instrum., vol. 82, no. 7, 074703, July 2011.

[12] W. Froncisz and J. S. Hyde, "The loop-gap resonator: a new microwave lumped circuit ESR sample structure," J. Magn. Reson., vol. 47, pp.515-521, 1982.

[13] T. Bandi, C. Affolderbach, C. Stefanucci, F. Merli, A. K. Skrivervik, and G. Mileti, " Compact High-Performance Continuous-Wave DoubleResonance Rubidium Standard With $1.4 \times 10^{-13} \tau^{-1 / 2}$ Stability", IEEETrans. Ultrason., Ferroelectr., Freq. Control, vol. 61, no. 11, pp. $1769-1778$, Nov. 2014.

[14] R. R. Mett, W. Froncisz, and J. S. Hyde, "Axially uniform resonant cavity modes for potential use in electron paramagnetic resonance spectroscopy “, Rev. Sci. Instrum. 72, 4188, 2001.

[15] A. Ivanov, T. Bandi, Guan-Xiang Du, A. Horsley, C. Affolderbach, P. Treutlein, G. Mileti, and A. K. Skrivervik. Experimental and Numerical Studies of the Microwave Field Distribution in a Compact MagnetronType Microwave Cavity. in proceedings 28th European Frequency and Time Forum (EFTF), Neuchatel, Switzerland, June 22-26, 2014.

[16] R. R. Mett, J. W. Sidabras and J. S. Hyde, "Uniform radio frequency fields in loop gap resonators for EPR spectroscopy", Appl. Magn. Reson. 31, 573589, 2007. 